\title{
Relaciones de género en la sociedad posmoderna: Percepciones y actitudes de estudiantes jóvenes sobre las relaciones de poder en las parejas
}

\author{
Gender Relations in Postmodern Society: Perceptions and Attitudes \\ of Young Students About Power Relations in Couples
}

\author{
María Jiménez-Delgado, Pablo de-Gracia-Soriano, Diana Jareño-Ruiz, \\ Mariano Agustín González-Chouciño'
}

\section{Resumen}

Este artículo presenta resultados de un proyecto que nace de la inquietud de un instituto público de Educación Secundaria Obligatoria (ESO) por conocer cómo son las relaciones de género entre su alumnado. El marco teórico que contribuye a contextualizar los resultados expuestos está centrado en las teorías del poder, del amor y los afectos, y del impacto de las TIC. El objetivo del estudio es diagnosticar cómo se expresan las relaciones de poder en las parejas en centros educativos. De esta manera, se espera poder obtener y plantear elementos que permitan a los centros generar estrategias para la acción futura a partir de los marcos emocionales de las relaciones de poder en las parejas. Con este fin se ha diseñado una herramienta cuantitativa que contribuye a la evaluación de cómo son estas relaciones. El cuestionario ha sido aplicado a una muestra de 140 estudiantes de tercero y cuarto de ESO con edades comprendidas entre los 14 y los 18 años. Posteriormente se ha realizado un análisis comparativo con otra muestra de 192 estudiantes de primer y segundo curso de Grado de Universidad, con edades comprendidas fundamentalmente entre los 18 y 20 años, con el fin de incrementar el rango de edad y valorar mejor la experiencia. El análisis de los datos obtenidos evidencia la existencia de diferencias estadísticamente significativas por edad, sexo y experiencia en pareja, así como la importancia de la comunicación para el proceso cotidiano de las relaciones afectivo-sexuales. Tras los análisis e interpretación de los datos se concluye que las relaciones de poder en las parejas están enmascaradas debido a una concepción romántica de las mismas.

\section{Palabras clave}

Relaciones de poder, relaciones de pareja, desarrollo afectivo, educación, género, juventud.

\section{Abstract}

This article presents results of a project that arises from the concern of a public institute of Compulsory Secondary Education (ESO) for knowing how gender relations are among its students. The theoretical framework that contributes to contextualize the exposed results is centered on the theories of power, love and affections, and the impact of ICT. The aim of the study is to diagnose how power relations are expressed in couples in educational centers. In this way, it is expected to be able to obtain and propose elements that allow the centers to generate strategies for future action based on the emotional frameworks of power relations in couples. To this end, a quantitative tool has been designed that contributes to the evaluation of how these relationships are. The questionnaire has been applied to a sample of 140 third and fourth students of ESO with ages between 14 and 18 years. Subsequently, a comparative analysis was carried out with another sample of 192 first and second year undergraduate students, aged between 18 and 20 years, with the aim of increasing the age range and valuing the experience better. The analysis of the data obtained evidences the existence of statistically significant differences by age, sex and couple experience, as well as the importance of communication for the daily process of affective-sexual relationships. After the analysis and interpretation of the data, it is concluded that power relations in couples are masked due to a romantic conception of them.

Keywords

Power relationship, Partnership, Emotional Development, Education, Gender, Youth.

\footnotetext{
1 María Jiménez-Delgado, Universidad de Alicante, maria.jimenez@ua.es; Pablo de-Gracia-Soriano, Universidad Complutense de Madrid y Universidad de Alicante, pablo.dgsoriano@ua.es; Diana Jareño-Ruiz, Universidad de Alicante, diana.jareno@ua.es; Mariano Agustín González-Chouciño, Universidad de Alicante, magc22@ alu.ua.es.
} 


\section{Relaciones de pareja y de poder entre la juventud: un análisis sociológico}

Entre los estudios sociológicos pioneros que incorporan la cuestión del poder en las relaciones afectivosexuales destacan los de Connell (1987, 2002, 2003, 2015), socióloga con una dilatada trayectoria personal e investigadora en los estudios de género. Connell (2003) considera el género como un sistema dinámico definido por relaciones de poder dentro de una determinada estructura social, que espera comportamientos distintos y asigna roles diferenciados a mujeres y hombres. En estas relaciones juega un papel central la noción de masculinidad hegemónica, por otra parte, cada vez más cuestionada, pues si bien sigue existiendo una jerarquía de masculinidades, éstas están en proceso de cambio.

Así mismo, una revisión de la literatura científica más amplia permite incorporar análisis sociológicos muy útiles para interpretar las actuales relaciones de pareja entre adolescentes y jóvenes a partir del estudio de la construcción identitaria en los procesos de socialización en las sociedades modernas y postmodernas (Beck y Beck-Gernsheim, 2001; Giddens, 2004; Castells y Subirats, 2007), así como a partir de trabajos que analizan los interesantes vínculos entre las relaciones afectivo-sexuales y los cambios culturales en el capitalismo. Pese a ello, el análisis de las relaciones afectivo-sexuales, tal y como ha apuntado la socióloga Mar Venegas (2011), no ha sido tratado desde la sociología de forma tan extensa como lo ha sido desde la psicología, especialmente desde la psicología social. No obstante, resulta imprescindible ampliar la mirada e investigar las relaciones y los cambios sociales y culturales que reproducen y perpetúan el patriarcado, así como los que están provocando una mayor equidad, libertad de elección y emancipación.

A Eva Illouz $(2007,2009,2012,2014)$ se le atribuyen algunas de las contribuciones más relevantes en este campo, que ayudan enormemente a entender desde explicaciones sociológicas «por qué -y cómo- duele el amon» (haciendo alusión al título de una de sus obras), qué tipo de relaciones queremos y buscamos en los contextos contemporáneos, cómo sentimos, en qué consiste la utopía amorosa en una sociedad de consumo o por qué los jóvenes y menos jóvenes pueden sentirse fascinados por un tipo de novela erótica popularizada en la última década, que en algunas ocasiones es llevada a la gran pantalla, como en el caso del libro escrito por la autora Erika Leonard Mitchell (2012), Cincuenta sombras de Grey (autora más conocida por el pseudónimo E. L. James). Este tipo de literatura parece identificarse con una nueva forma de amor romántico que como bien explica Illouz viene a legitimar nuevamente las relaciones de poder entre hombres y mujeres.

Desde un punto de vista académico, más centrado en el tema concreto que nos ocupa en esta investigación, esto es, las relaciones de pareja en la adolescencia y juventud, destacamos uno de los trabajos más recientes en España desde la perspectiva sociológica, «El romance adolescente. Un análisis sociológico de la política afectivosexual en la adolescencia» de Mar Venegas (2018). Dicho estudio resulta especialmente útil para explicar las relaciones de pareja como relaciones mediadas por el poder y condicionadas por factores como la clase social, el género y la etnia.

El ciclo vital de todo ser humano lleva consigo los procesos de socialización y de construcción identitaria. Desde que nacemos, percibimos y experimentamos el mundo que nos rodea, y nuestra identidad se conforma a medida que nos relacionamos con otras personas. En este proceso de interacción y en sus rituales asociados (Collins, 2009), es donde surgen, se aprenden y modifican los sentidos, significados, normas, valores e instituciones, que interiorizan los individuos y se reconfiguran a partir de las experiencias vitales y sus contextos. 
La identidad, término que se ha empezado a utilizar de forma intensa desde hace medio siglo, no remite -al menos no de forma absoluta- a la historia ni a nuestras raíces, sino a la subjetividad y a la producción de sentido (Kaufmann, 2015). El proceso de construcción identitario es dinámico y cambiante (Sobczyk, 2015), por tanto, no es una esencia, una entidad homogénea y cerrada, y está condicionado por la cultura o las culturas en las que está inmerso el individuo, que son siempre dinámicas. Los roles de género están en proceso de cambio social, siendo muchos los factores que los condicionan, así como también están cambiando las relaciones de pareja y de género. Es en este ámbito en el que la sociología hace aportaciones imprescindibles (Connell, 2002; Illouz, 2007, 2009, 2012, 2014).

Resulta cuanto menos paradójico seguir analizando y constatando las relaciones de poder y de dominación entre hombres y mujeres en sociedades democráticas y libres, muy distintas a las que en su día analizó Bourdieu (2005), y resulta mucho más inquietante cuando nos referimos a la población adolescente y joven, una generación que ha nacido y vive en un mundo más abierto y plural que el de sus progenitores.

Como demuestran diversos estudios (Rubio, 2015), las relaciones de pareja entre la población más joven pueden ser, en algunos aspectos, menos democráticas y libres de lo que se hubieran podido imaginar las generaciones que les preceden, especialmente quienes lucharon desde los movimientos feministas por la igualdad de derechos entre hombres y mujeres, tanto en el espacio público como en el privado, así como por el reconocimiento de la diversidad de opciones sexuales (Cruz y Cobo, 1991). Si bien en el espacio público, en las diversas instituciones sociales y educativas, los avances en materia de igualdad son evidentes, es en el espacio más privado donde las relaciones de poder y de control siguen existiendo. El uso de las redes sociales como forma de comunicación cotidiana, especialmente entre los jóvenes, puede estar generando nuevas formas de control en las relaciones de pareja permitiendo formas de dominación sutiles, pero más totalitarias que las tradicionales que estaban limitadas a un espacio y un tiempo concretos.

Es en esta sociedad tecnológica y de la comunicación en la que las relaciones de pareja entre los jóvenes son simultáneamente privadas y públicas (Cruz y Cobo, 1991; Innerarity, 2006), y en la que lo más íntimo puede difundirse, globalizarse y «viralizarse», donde pueden generarse nuevas relaciones de (in) dependencia, de (des)confianza y de control o de autonomía.

\section{La relación de la población joven y adolescente con las TIC}

Los smartphones y las redes digitales han tenido un profundo impacto en las nuevas generaciones, pasando a formar parte de la vida cotidiana de la mayoría de la población, convirtiéndose en un instrumento que supera el ámbito laboral y comunicativo (Blanco, 2014). También construyen nuevas pautas culturales a partir de las innumerables posibilidades que ofrecen las TIC en la recreación de encuentros y relaciones virtuales románticas (Illouz, 2007). Internet y las redes digitales han cambiado la lógica de las relaciones sociales que tienden hacia una mayor fragilidad, flexibilidad y temporalidad de los vínculos (Bauman, 2003; Muñiz et al., 2015; Turkle, 2017). No obstante, las relaciones que se crean en el mundo online, derivan de las preexistentes en el contexto offline. En este sentido, se da una desaparición de la dicotomía virtual versus real, al no poder distinguirla, así como también de la línea que separa lo público, lo privado y lo íntimo, puesto que se tiende a volcar una gran cantidad de información en este tipo de redes, ya sea consciente o inconscientemente (Giddens, 2004; Innerarity, 2006; Muñiz et al., 2015).

En el caso de la población joven y adolescente, el teléfono móvil se ha convertido en un bien personal de enorme importancia en sus vidas, considerándose un elemento fundamental en la construcción de su 
identidad individual (Jiménez-Albiar et al., 2012). Se trata de un símbolo de estatus cuya ausencia es capaz de generar sentimientos negativos y problemas de autoestima. La importancia del teléfono móvil es abordada por diversos autores, específicamente Velázquez (2011), quien asevera que se puede encontrar una explicación teórica a este suceso siguiendo los planteamientos de Heidegger, en los que deja constancia de cómo la técnica es la que domina al individuo y no al contrario. Walter Benjamin (1973) llama a esto noción de insubordinación de la técnica, debido a que la técnica produce fascinación y esta deviene en aceptación ciega, sin importar el control y la dominación que ejerce sobre nosotros dicha tecnología. Se trata de un efecto totalizador del teléfono móvil, el cual confirman Bringué y Sádaba (2009). En las conclusiones de su estudio afirman que el grado de penetración del teléfono móvil entre la «generación interactiva [...] hace que sea una pantalla difícil de apagar» (Bringué y Sádaba, 2009: 177).

Las cuestiones expuestas tienen especial relevancia en relación al entorno educativo y familiar, puesto que los y las jóvenes consideran la red como una extensión del mundo real, mientras que este se concibe como un sitio distinto para los padres, las madres y el profesorado (Busquet y Uribe, 2011). Se da una contradicción en el marco educativo, puesto que el ordenador y el uso de Internet son, con frecuencia, terreno donde las figuras maternas y paternas reciben instrucciones de sus hijos e hijas (Díaz-Granados, 2007). El contexto familiar y educativo, por tanto, se convierte en un escenario de confrontación entre dos generaciones que se entienden lejanas respecto a estas tecnologías.

Las primeras relaciones de pareja en la adolescencia están totalmente mediadas por la tecnología móvil y, por tanto, están condicionadas igualmente por las relaciones de poder que se construyen de este modo.

\section{Sexismo como relación de poder y sus manifestaciones en las relaciones de pareja} El sexismo, identificado como aquella actitud discriminatoria de quien infravalora a las personas de distinto sexo o hace distinción de las personas según su sexo, se manifiesta de diferentes formas (De La Peña et al., 2011). La revisión de la literatura muestra una mayor atención en la actitud de superación de los varones respecto a las mujeres. Existe un sexismo hostil o tradicional, que hace referencia a las actitudes de prejuicio o conductas discriminatorias basadas en una supuesta superioridad del hombre sobre la mujer. Este tipo genera, según los autores, un esquema de tres elementos: el paternalismo dominador, la diferencia de género competitiva y la hostilidad heterosexual. Por otra parte, destacan un segundo tipo de sexismo, el benévolo. Esta categoría refleja una apariencia más respetuosa y sutil, donde se identifican también tres elementos: el paternalismo protector, la diferencia de género complementaria y la intimidad heterosexual. Estos elementos crean un sistema de refuerzos y castigos destinado a hacer saber al grupo subordinado como ha de comportarse. Ambas formas de sexismo conforman el llamado «sexismo ambivalente», tal y como se define en el trabajo de De la Peña y sus colaboradores (2001). De esta forma, el sexismo se convierte para estos autores en un hecho que es interiorizado por los sujetos, tanto en sus roles y rasgos, como en su proceso de legitimación de la autoridad según el sexo en las parejas. Esta situación se da mediante diferentes estrategias de coacción: aislamiento, desvalorización, manifestación de creencias sexistas, violencia física o abuso sexual, entre otras.

La unión conceptual generada entre el amor romántico y el sexismo genera un escenario en el cual, a través del proceso de idealización propio de esta forma de amor, se toleran o justifican actitudes y comportamientos relacionados con la violencia de géneros (Blanco, 2014). En esta línea, el tipo de relación de pareja, la autoestima y el comportamiento sexista se muestran correlacionados de forma directamente 
proporcional. Además, las actitudes y comportamientos sexistas se refuerzan con las TIC, debido a las posibilidades que estas tecnologías ofrecen para realizar acciones violentas o de acoso (Muñiz et al., 2015).

A partir de este marco teórico se aborda el trabajo de investigación que aquí se presenta.

\section{Objetivos y metodología}

Esta investigación parte de una inquietud del equipo directivo y del profesorado del Departamento de Orientación de un Instituto de Educación Secundaria (IES) de la ciudad de Alicante. Esta inquietud apunta hacia la percepción, por parte del profesorado, de un repunte de comportamientos machistas en el aula, agravados en y por el uso de plataformas digitales. Es por ello que desde el centro se requiere un diagnóstico de la situación que permita generar estrategias socioeducativas de acción en los marcos de igualdad y convivencia. El objetivo principal de este trabajo es, entonces, conocer qué actitudes tiene el alumnado respecto a las relaciones de poder en las parejas.

El marco teórico desvela la importancia de contemplar la perspectiva de género y el impacto de las TIC en las relaciones de pareja, elementos que se han tenido en cuenta en el proceso de diseño de la investigación. Este contexto, unido al carácter cooperativo del proyecto con el centro demandante, propició la elección de un diseño cuantitativo basado en la adaptación de una encuesta realizada desde la Universidad de Santiago (Rodríguez y Vázquez-Portomeñe, 2015), dado que desde el centro se manifestó la necesidad de medir la posible gravedad del problema. La adaptación se llevó a cabo a partir de la revisión de la literatura científica y de la discusión de cada ítem del cuestionario base por parte de los miembros del equipo investigador. Este proceso se materializó en un nuevo cuestionario que cuenta con cincuenta y cinco preguntas: cuarenta y siete ítems sobre el grado de acuerdo o desacuerdo con cada uno de ellos y ocho preguntas de carácter sociodemográfico. El instrumento se sometió a la fase de pretest contando con diez alumnos y alumnas y tres profesionales ajenos al equipo investigador, quienes contribuyeron a dialogar de forma crítica con el grupo para adaptar tanto la estructura del cuestionario como la revisión de los ítems.

El cuestionario se diseñó de forma que pudiera ser autoadministrado por personas con catorce años y más, puesto que las dinámicas y condiciones del centro favorecían este tipo de procedimiento. Así, es el alumnado quien responde a las preguntas del cuestionario, y el personal docente, formado mediante sesiones formativas (briefing) por el equipo investigador, representa en el aula la figura de apoyo y consulta. Estas sesiones formativas consistieron en reuniones con el profesorado, previas al momento de ejecución del campo, en las que se explicaron los objetivos y contextos del proyecto, así como la necesidad de generar un clima de calma en el aula con el objetivo de reducir las interferencias que pudieran producirse entre el alumnado, y quizá, por tanto, en sus respuestas. También se comentaron de forma pormenorizada todas las preguntas del cuestionario y se dieron algunas pautas de cómo resolver posibles dudas que pudieran surgir, relacionadas con la comprensión de alguna pregunta o la cumplimentación del cuestionario. Sobre este último aspecto se señaló la importancia del orden de las preguntas, así como recordar no poner el nombre en la hoja para garantizar el anonimato, etc. Sobre el primer aspecto se recomendó que se tratara de explicar con otras palabras el enunciado, sin perder el sentido del argumento, y en caso de no hacerse viable esta opción, que se aceptara la no respuesta y continuar con otra pregunta.

La fase de trabajo de campo supuso que el equipo reflexionara acerca de la posibilidad de ampliar el proyecto e incorporar a población universitaria, con el objetivo de poder abarcar un rango mayor de edades, permitiendo la comparación de muestras, al menos, por sexo, edad y centro de pertenencia, dado 
que el calendario académico del centro impidió aplicar el cuestionario en otros cursos, limitando el rango de edad. Así, además de los casos del Instituto de Educación Secundaria, contamos con casos de tres Facultades diferentes de la Universidad de Alicante (Facultad de Ciencias Económicas y Empresariales, Facultad de Derecho y Facultad de Educación), siendo estas seleccionadas por una mayor accesibilidad del equipo de investigación.

En el caso del instituto, el universo de análisis fueron 162 alumnas y alumnos matriculados en el centro en los cursos de $3^{\circ}$ y $4^{\circ}$ de ESO. La elección de los cursos se dio por petición del centro, dado que era en estos donde el centro identificaba la presencia de mayores riesgos en las relaciones de género entre estudiantes. Aunque el planteamiento inicial era poder obtener respuesta de todo el censo, finalmente se obtuvo una muestra que representa al 86,4\% (140 personas), con edades comprendidas entre los 14 y los 18 años, teniendo el 74\% de los casos entre 15 y 16 años. En el segundo caso, la muestra universitaria fue de 192 alumnas y alumnos de primer curso de los Grados en Relaciones Laborales y Recursos Humanos, Maestro en Educación Primaria, Sociología y en el segundo curso del Doble Grado en Derecho y Criminología, con edades comprendidas entre los 18 y 40 años, teniendo el 84,5\% de los casos entre 18 y 20 años. La elección de los cursos está motivada por las edades más cercanas a la muestra del IES, así como por acceso directo de los miembros de equipo investigador. En total se generó una matriz que cuenta con 332 casos.

Para captar los matices de la edad y poder trabajar con la variable de forma operativa, ésta fue recodificada para agruparlas en 5 categorías: «juventud en pubertad» (14 y 15 años), «juventud adolescente menor de edad》 (16 y 17 años), «juventud adolescente mayor de edad》 (entre 18 y 20 años), «juventud》 (entre 21 y 24 años) y «mayores de 24 años». Si bien la variable edad en el conjunto de ambas muestras varía entre los 14 y los 40 años, dicha variable se encuentra, en el 92,2\% de los casos, entre los 14 y los 21 años.

Para la sistematización de toda la información se utilizó el software SPSS, utilizando el análisis estadístico como procedimiento de tratamiento de los datos. En el apartado de resultados se muestran análisis univariables y bivariables de las cincuenta y cinco preguntas que contiene el cuestionario.

Por tanto, la investigación se ha planteado con carácter exploratorio. El principal objetivo es desarrollar un diagnóstico en relación a las cuestiones planteadas con dicho centro educativo. Debido al contexto de origen del proyecto, la investigación carece de hipótesis que guíe la obtención de resultados, aunque los que se obtengan de este trabajo servirán para el planteamiento de nuevas investigaciones e hipótesis sobre la situación actual de las relaciones entre dichos grupos de edad en los centros educativos.

\section{Análisis de resultados sociodemográficos de la muestra}

La estructura de la muestra del IES se compone de 140 casos, siendo un 53,5\% mujeres y el 45\% hombres $(2,5 \%$ de no respuesta). Dos tercios de la población encuestada manifiesta haber tenido pareja alguna vez, siendo un 78,2\% entre los hombres y un 50,8\% entre las mujeres. En el caso de la orientación sexual, un $12,9 \%$ de los casos manifiestan tener otra orientación diferente a la heterosexual (mayoritariamente bisexuales), siendo en un 77,8\% de los casos mujeres. Además, un 64,8\% de quienes se manifiestan heterosexuales afirman haber tenido pareja frente a un $72,2 \%$ en el caso de otras orientaciones sexuales.

La edad resulta una variable importante. Así, entre los «jóvenes en pubertad» un 29,6\% manifiestan no haber tenido pareja frente a un 16,1\% entre los adolescentes mayores y menores de edad. Podemos apreciar como el $72,2 \%$ de las personas que manifiestan tener otra orientación sexual diferente a la heterosexual 
se encuentran dentro de la categoría de edad «juventud en pubertad», frente al 27,8\% de los casos restantes, localizados en las categorías de «adolescencia» (es decir, entre 16 y 20 años).

En cuanto al origen, un $77 \%$ de los casos manifiestan ser de origen español, mientras que un 12,9\% procede de América del Sur, un 5\% de Europa de la UE y el resto de otras regiones. La población latinoamericana es el grupo que tiene un peso más elevado de personas que han estado en pareja, obteniendo un porcentaje del 72,2\%, mientras que los españoles se sitúan en segunda posición con un $61,7 \%$.

En relación a la muestra universitaria, encontramos un total de 192 cuestionarios válidos, siendo un $69,3 \%$ mujeres y un 30,7\% hombres. En este caso hemos de eliminar el valor analítico de las variables «edad»y «origen», puesto que no hay suficientes casos representativos (un 5,6\% proceden de fuera de España). El 87\% de los casos manifiestan haber tenido pareja, no existiendo diferencias significativas por sexo ni orientación sexual. Por otra parte, resulta destacable cómo, si bien un 7,8\% de las personas encuestadas manifiestan tener una orientación sexual diferente a la heterosexual, de este porcentaje un $80 \%$ son mujeres.

\section{Análisis de valores extremos}

Este apartado pretende dar a conocer cuáles son los ítems con los que la población entrevistada está más de acuerdo y más en desacuerdo, es decir, cuáles son los ítems que adquieren los valores más extremos en las respuestas, ya sea a favor o en contra. Para ello, se ha procedido a dicotomizar las variables del cuestionario a partir de las cuatro categorías iniciales (totalmente de acuerdo, bastante de acuerdo, poco de acuerdo, nada de acuerdo).

A continuación, se ha calculado la diferencia entre los valores de acuerdo y desacuerdo que han surgido de las frecuencias de las variables recodificadas, con el propósito de averiguar si el balance que dan los alumnos y alumnas a cada ítem es positivo o negativo. Si el resultado ha sido negativo, significa que se está poco de acuerdo con lo que el ítem afirma, siendo mayor el grado de desacuerdo cuanto menor sea este número. Por el contrario, si el número resultante de la operación es positivo, significa que se está de acuerdo con lo que enuncia el ítem, aumentando también el grado de acuerdo cuanto mayor sea la cifra obtenida.

En relación al análisis de los ítems con los que se está más y menos de acuerdo, en el caso de la muestra del IES, se evidencia que los ítems con los que más de acuerdo se está son los relacionados con la igualdad de opinión («mis opiniones son tan importantes como las de mi pareja»), la comunicación en la pareja («nuestra relación se basa en la comunicación. Nos escuchamos»), la sinceridad («no tener secretos es la base de la confianza y el respeto, en una relación de pareja», «una buena relación de pareja se basa en la comunicación»), y la protección («si alguien se pasa conmigo, mi pareja me defiende aunque no se lo pida»). En cuanto a los ítems con los que se está más en desacuerdo, estos están centrados fundamentalmente en el rechazo hacia el carácter humillante y controlador en las parejas ( veces me siento humillada/ o por compañeros/as del sexo contrario», «mi pareja se burla de míy me avergüienza cuando estamos con más gente», «me siento bumillada/ o por mi pareja», «bago caso a mi pareja en todo lo que me dice, porque sabe más que yo», «confío más en mi pareja si me da las claves de sus redes sociales»).

En el caso de la muestra universitaria, observamos que los ítems son prácticamente los mismos que en la muestra del IES; no obstante, el grado de desacuerdo o de acuerdo en cada caso es mucho mayor en el grupo de universidad. El primero de estos ítems es «me siento más tranquilo y confío más en mi pareja si me da las contraseñas de sus redes socialess, el cual aparece como extremo negativo en ambas muestras, aunque de forma más acentuada en la universitaria. En el caso del ítem «a veces me siento humillado/a por compañeros/as del sexo 
contrario», aparece como extremo en la muestra del IES pero no en la de universidad. Por último, el ítem que presenta valores más extremos entre el alumnado de educación obligatoria es «mi pareja se burla de míy me avergüienza cuando estamos con más gente»; mientras que en la muestra universitaria encontramos «mi pareja se burla de mí y me avergüenza cuando estamos a solas». En ambos casos, se trata de resultados que muestran estar en desacuerdo con tales afirmaciones. El análisis de los anteriores ítems parece apuntar a que a edades más tempranas se hace más relevante la búsqueda activa de aceptación sobre determinados grupos sociales y, por tanto, es más importante la imagen «pública» que se pueda tener y ofrecer. No obstante, como se muestra en el siguiente apartado, esta lectura de los datos se vuelve ambivalente, pues también puede interpretarse que, a mayor edad, mayor conciencia puede haber sobre lo socialmente deseable. Así mismo esa imagen está muy condicionada por el uso y el control que se ejerce a través del teléfono móvil y de las redes sociales (Bringué y Sádaba, 2009) como una nueva forma de consumo emocional (Illouz, 2009, 2012) que influye enormemente en el tipo de control y de ejercicio del poder en la pareja.

El análisis de las etiquetas de las variables que obtuvieron valores extremos en ambas muestras reveló un conjunto de categorías y conceptos que ofrecen una visión inicial de cuáles son las áreas que aparecen como destacadas por la juventud estudiada. Tal y como se recoge en la Figura I, son las siguientes: protección, libertad, igualdad, comunicación, confianza, obediencia, maltrato.

Figura I. Nube de categorías surgidas de los valores extremos

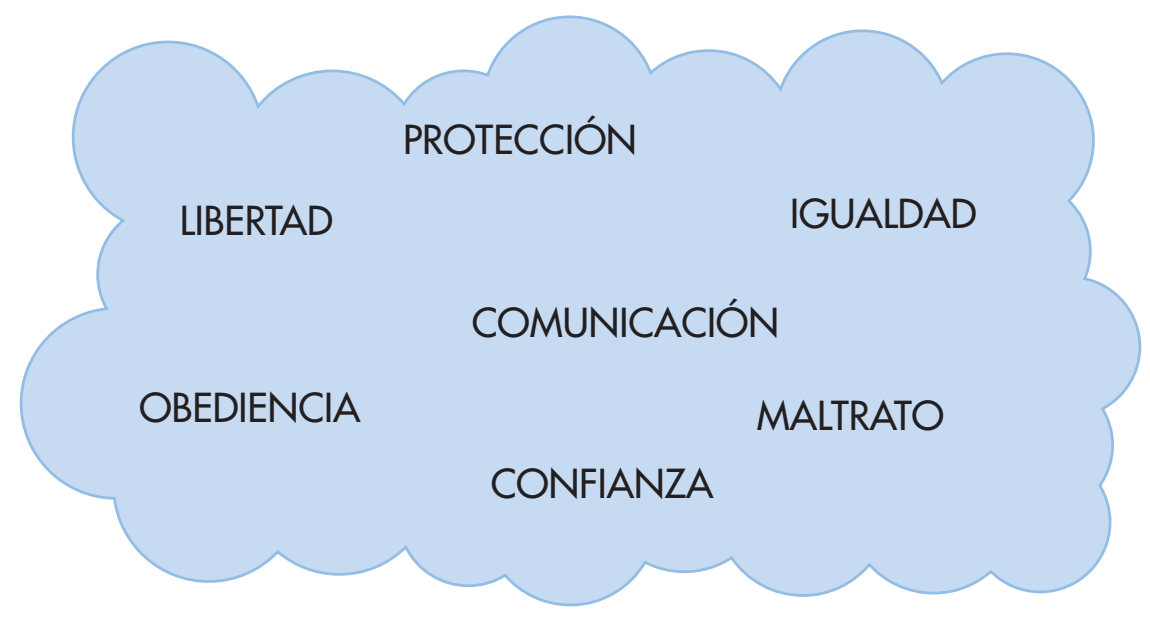

Fuente: elaboración propia.

\section{Análisis por dimensiones}

Puesto que el cuestionario con el que se ha trabajado es el resultado de una adaptación de otro a partir de la literatura científica revisada, la investigación ha requerido incluir un proceso de categorización de los ítems para alcanzar el concepto objeto de estudio: las relaciones de poder en las parejas. En el proceso normal de una investigación, lo habitual habría sido desgranar el concepto en dimensiones y posteriormente definir qué variables se piensa (hipótesis) o se conoce (revisión de literatura) que serán las que definan a cada una de las dimensiones. En este caso, al tratarse de una adaptación de un cuestionario ya elaborado por otro equipo, se ha hecho necesario un proceso inverso de categorización y formación de dimensiones, a partir de las variables del cuestionario. De esta manera, se clasificó cada ítem en la temática más cercana que contiene. El proceso finalizó con la obtención de cinco dimensiones que, para esta investigación, se han resuelto dotadoras de significado para definir el tipo de relación de pareja que tiene la juventud (Figura II). 


\section{Figura II. Dimensiones ad hoc de las relaciones de poder en las parejas}

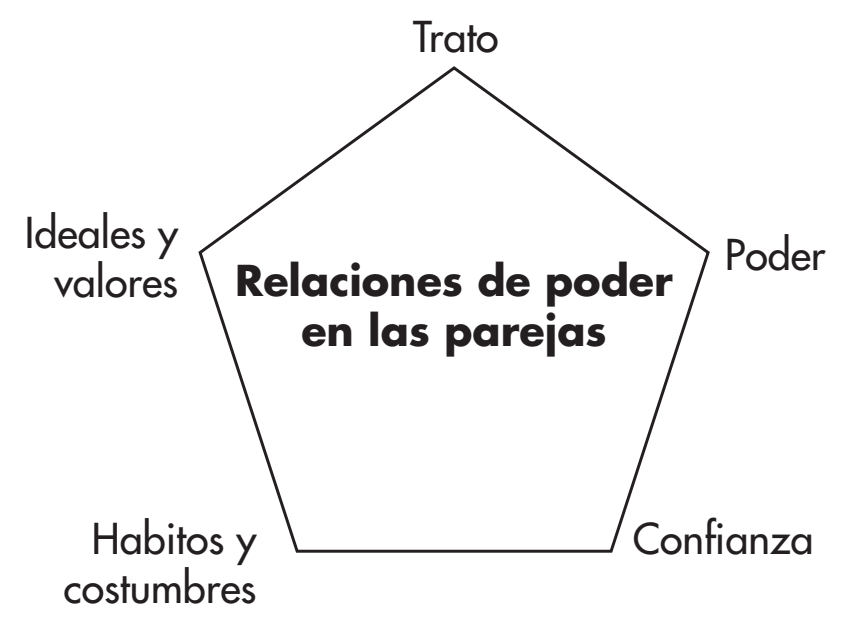

Fuente: elaboración propia.

Se debe advertir que, de forma trasversal a todas las categorías analizadas, se puede apreciar una mayor homogeneidad al responder por parte de los universitarios en comparación con la muestra de estudiantes de educación secundaria. Esto se traduce en que los valores que adquieren la mayoría de ítems resultan más extremos en un sentido u otro en la población universitaria.

Confianza: La categoría de confianza recoge siete de los ítems del cuestionario, y es la dimensión en la que se da el cambio más significativo a la hora de comparar ambas muestras. Así, la reducción en las variables negativas es casi total, dándose porcentajes «de acuerdo» inferiores al 5\%. El grado de acuerdo con la cesión de contraseñas de las redes sociales con la pareja («No tengo problema en darle las contraseñas de mis redes sociales a mi pareja, si asi se siente más tranquilo/ a y confía más en mî), muestra unas diferencias significativas entre ambas muestras, posicionándose la población universitaria mucho más en contra de este ítem. También hay diferencias significativas en cuanto a la privacidad de las conversaciones en las redes sociales («No tengo problema en enseñar mis conversaciones de Whats App y redes sociales a mi pareja, si asi se siente más tranquila/ o conmigo»), reduciéndose en un tercio el grado de acuerdo entre los universitarios en comparación a la muestra del alumnado del instituto.

Hábitos y costumbres: Esta dimensión es el resultado de cinco de los ítems del cuestionario. En este caso, se observa la misma estructura en los resultados para ambas muestras, así como valores similares. Sin embargo, en el caso de la muestra universitaria el ítem «nuestra relación se basa en la comunicación» se incrementa en torno a un $20 \%$ en su grado de acuerdo. Atendiendo a los datos, la población universitaria parece valorar y practicar en mayor medida la comunicación con sus parejas.

Trato: En este caso, la dimensión es el resultado de 12 ítems. El análisis de ésta arroja la misma tendencia general expuesta anteriormente. Casi todos los enunciados que se refieren a la dimensión de trato muestran una diferencia significativa entre las dos muestras, siendo en la universitaria aquella en la que se obtienen valores de desacuerdo mayores. El ítem que mantiene una estructura de respuestas más similar es el de «Mi pareja me dice con frecuencia que con mi actitud le estoy provocando para que se enfade». El grado de acuerdo con esta afirmación se encuentra entre el 11,2\% (universidad) y el 17,9\% (IES). Por el contrario, el conjunto de enunciados en el que se muestran mayores diferencias (grados de acuerdo inferiores al $2 \%$ ) entre ambos centros hacen referencia al desprecio de un miembro hacia el otro ( $A$ veces le digo a mi pareja que no vale nada»; «A veces mi pareja dice que no valgo nada, que no sé hacer nada»; «Mi pareja se burla de mí y me avergüenza cuando estamos con más gente»; «Mi pareja se burla de mí y me avergüenza cuando estamos a solas»). 
Ideales y valores: En esta dimensión, con diez ítems, se destaca de forma generalizada una visión de libertad, independencia e igualdad en la pareja, lo cual se materializa en la totalidad de las respuestas, tratándose de un elemento estructural en lo que a esta dimensión se refiere. No obstante, cabe destacar el ítem «si alguien se pasa conmigo, espero que mi pareja me defienda», dándose una diferencia del $5 \%$ en el grado de acuerdo entre ambas muestras (40\% entre los universitarios y $35 \%$ en la muestra del IES).

Poder: La dimensión de poder se ha construido a partir de 13 ítems, de los que resulta destacable que para ambas muestras se obtienen resultados similares en los siguientes ítems: «Mi pareja dice que no puede estar sin mì, «Si alguien se pasa conmigo, mi pareja me defiende, aunque no se lo pido» $\mathrm{y}$ «Necesito estar en constante comunicación con mi pareja, aunque sea por Whats App». Se muestra, por tanto, una situación de dependencia basada en la necesidad del uno por el otro, así como la necesidad de una comunicación constante. Los datos señalan también que en estas relaciones se espera ser protegida/o por la pareja. Dicho de otra forma, existe una 'protección esperada' en las relaciones de pareja.

\section{Análisis de desigualdades entre las categorías de las variables sociodemográficas}

Es importante conocer si hay percepciones y actitudes que muestran cierta desigualdad entre las categorías de las variables sociodemográficas. Este análisis permite conocer, por tanto, si piensan igual (para cada uno de los ítems) hombres y mujeres; heterosexuales y otras orientaciones (los pocos casos relativos de otras orientaciones se tradujeron en una agrupación de las mismas); quiénes han tenido pareja y quiénes no; adolescentes y jóvenes; la población nacida en España y la nacida en otros países. Para ello se ha realizado la prueba $Z$ entre cada una de las variables sociodemográficas y cada ítem del cuestionario. El análisis, además, se ha realizado para cada una de las muestras y para el conjunto de las mismas, con el objetivo de extraer las principales diferencias que existen a nivel comparativo y global.

El primer paso, necesario para aplicar esta prueba, ha consistido en recodificar cada una de las variables en variables dicotómicas. Como en el análisis realizado para conocer los valores extremos, los ítems del cuestionario se recodificaron en dos categorías de respuesta: de acuerdo o en desacuerdo. Por su parte, las variables de carácter sociodemográfico se dicotomizaron tal y como se expone en el primer párrafo de este apartado.

Los datos y análisis que se presentan en este apartado se limitan a aquellos casos en los que existen diferencias significativas y que, por tanto, requieren especial atención para conocer en qué aspectos existen actitudes diferentes -no necesariamente contrarias- entre los adolescentes y jóvenes asociados a cada una de las categorías de las variables sociodemográficas.

\section{a. Desigualdades en la muestra del instituto de educación secundaria}

La Tabla I recoge el resumen de los resultados significativos de la prueba $Z$ para la muestra del IES. Este análisis revela que las variables más significativas a la hora de explicar las diferencias entre las categorías son las variables «sexo» y «baber tenido pareja». De esta forma, se observa en los chicos una tendencia mayor a aceptar cuestiones relacionadas fundamentalmente con las dimensiones de la confianza y los ideales y valores, así como más concretamente con ítems relacionados con el control en la pareja.

La pregunta P19 merece especial mención, debido a que es un ítem que muestra cómo los hombres no esperan que sus parejas les defiendan, mientras que las mujeres se muestran divididas respecto a este ítem, habiendo tantas que están de acuerdo como las que no lo están. Este es un caso claro que muestra que, para esta variable, hombres y mujeres no piensan igual, aunque no quiere decir que lo 
hagan de forma contraria. La desigualdad se encuentra, precisamente, en que la mayoría de los hombres $(68,5 \%)$ no esperan ser defendidos por su pareja, mientras que hay tantas mujeres que lo esperan como las que no. De acuerdo con el concepto de relaciones de poder en la pareja definido por Connell (2003, 2005), hay una construcción de roles de género que asigna mayor dependencia de las mujeres respecto de los varones, construyendo así una específica relación de poder que se confirma en el análisis.

Respecto a la variable edad, se observa que la población más joven de la muestra manifiesta diferencias significativas respecto a los más mayores en cuanto al ítem referido al control en la pareja: «Si cuando mi pareja me ve en línea en Whats App, me pregunta con quién hablo, es porque se interesa por mír. En este caso, aunque ambos grupos de edad están mayoritariamente en desacuerdo con el enunciado de la pregunta, la prueba $Z$ revela que existen diferencias estadísticamente significativas. En tanto que más del $90 \%$ de los más jóvenes están en desacuerdo (el 9,2\% está de acuerdo), tres cuartas partes del alumnado más mayor manifiesta estarlo también.

Por último, aparecen diferencias entre quienes no han tenido y quienes sí han tenido pareja, y estas diferencias se muestran en dos ítems que hacen referencia a aspectos de comunicación en la pareja (ver Tabla I). En ambos ítems se muestra que quienes han tenido o tienen pareja tienen un grado de acuerdo significativamente mayor que quienes no han tenido. De estos ítems se puede deducir que uno de los elementos que se refuerzan con la experiencia en pareja (ya que en ambos ítems los dos grupos están mayoritariamente de acuerdo con la afirmación), es la importancia de la comunicación.

Tabla I. Resultados del análisis de desigualdades para la muestra del IES

\begin{tabular}{|c|c|c|}
\hline VARIABLE & ÍTEMS & RESULTADO \\
\hline \multirow{4}{*}{ Sexo } & $\begin{array}{l}\text { P8. «Si un miembro de la pareja se preocupa constantemente por saber que está haciendo el } \\
\text { otro, es una muestra de afecto e interés». }\end{array}$ & $\begin{array}{l}\text { Mayor grado de acuerdo entre los hombres. (40\% frente al } \\
22 \% \text { ) }\end{array}$ \\
\hline & $\begin{array}{l}\text { P18. «No tengo problemas en darle las contraseñas de mis redes sociales a mi pareja, si así se } \\
\text { siente más tranquilo/a y confía más en mi». }\end{array}$ & Mayor grado de desacuerdo entre las mujeres. $(82 \% \mid 65 \%)$ \\
\hline & P19. «Si alguien se pasa conmigo, espero que mi pareja me defienda». & Mayor grado de desacuerdo entre los hombres. (69\% | 50\%) \\
\hline & $\begin{array}{l}\text { P30. «No tengo problemas en enseñar mis conversaciones de WhatsApp y redes sociales a mi } \\
\text { pareja, si así se siente más tranquilo/a». }\end{array}$ & Mayor grado de acuerdo entre los hombres. (45\%|23\%) \\
\hline Edad & $\begin{array}{l}\text { P5. «Cuando me ve en línea en WhatsApp, me pregunta con quién hablo, es porque se interesa } \\
\text { por mí». }\end{array}$ & A menor edad, mayor desacuerdo. (91\%|76\%) \\
\hline \multirow{2}{*}{$\begin{array}{l}\text { Haber tenido } \\
\text { pareja }\end{array}$} & P.44. «Una buena relación de pareja se basa en la comunicación». & $\begin{array}{l}\text { Mayor grado de acuerdo entre quienes han tenido pareja. } \\
(93 \% \mid 73 \%)\end{array}$ \\
\hline & P.47. «Nuestra relación se basa en la comunicación». & $\begin{array}{l}\text { Mayor grado de acuerdo entre quienes han tenido pareja. } \\
(86 \% \mid 53 \%)\end{array}$ \\
\hline
\end{tabular}

Fuente: elaboración propia.

\section{b. Desigualdades en la muestra universitaria}

Los resultados de la prueba $Z$ para la muestra universitaria para cada una de las variables sociodemográficas, pone de manifiesto que solo el sexo se muestra como variable generadora de respuestas significativamente distintas entre hombres y mujeres. La mitad de los seis ítems que revelan considerarse de forma distinta según si se es hombre o mujer (Tabla II), coinciden con los resultados obtenidos en la muestra del instituto (P18, P30 y P48), siendo las mujeres universitarias las que están más en desacuerdo con estos ítems, los cuales hacen referencia a la ausencia de privacidad y a la cuestión del control en la relación de pareja. 
Respecto a los otros tres ítems, los resultados de la prueba $\mathrm{Z}$ indican que el $16 \%$ de los hombres considera que acaba haciendo lo que su pareja quiere cuando tienen planes distintos, siendo un $10 \%$ en el caso de las mujeres. Ésta es la variable con mayor proximidad entre sexos, mientras en las otras dos se obtienen diferencias de entre el 14\% y el 25\%. En el primer caso, un 14\% más de hombres que de mujeres manifiestan necesitar estar en constante comunicación con su pareja. En el segundo, un $25 \%$ más de hombres dice renunciar a hacer cosas que le apetecen para evitar conflictos.

\title{
Tabla II. Resultados del análisis de desigualdades para la muestra universitaria
}

P2. «Cuando no nos apetece el mismo plan a mi pareja y a mí, acabamos haciendo lo que mi pareja quiere». M. (90\% | 84\%)
P3. «Renuncio a hacer cosas que me apetecen para no tener conflictos con mi pareja».
M. $(95 \% \mid 70 \%)$

P12. «Necesito estar en constante comunicación con mi pareja, aunque sea por WhatsApp».

M. $(80 \% \mid 66 \%)$

Sexo

P18. «No tengo problema en darle las contraseñas de mis redes sociales a mi pareja, si así se siente más
tranquilo/a y confía más en mí».

P30. «No tengo problema en enseñar mis conversaciones de WhatsApp y redes sociales a mi pareja, si así se siente más tranquila/o y confía más en mí».

\author{
M. $(85 \% \mid 61 \%)$
}

P.48. «Cuando un miembro de la pareja corrige duramente al otro, lo hace para ayudarle a mejorar». $\quad$ M. (88\% | 59\%)

1 M: mujeres; H: hombres

Fuente: elaboración propia.

\section{Desigualdades comparadas entre muestras}

La prueba $\mathrm{Z}$ para la comparación de las posibles desigualdades entre las muestras de instituto y la universitaria revela la existencia de 20 ítems en los que el alumnado difiere significativamente en su manifestación del grado de acuerdo o desacuerdo. Los ítems donde se muestra mayores diferencias entre muestras se corresponden con las dimensiones de ideales y valores («Si un miembro de la pareja se preocupa constantemente por saber qué está haciendo el otro, es una muestra de afecto e interés», «Mis opiniones son tan importantes como las de mi pareja»), confianza («No tengo problema en darle las contraseñas de mis redes sociales a mi pareja, si asi se siente más tranquilo/ a y confia más en mì), trato («Mi pareja me dice que sería capaz de "cualquier cosa" si le dejara») y poder "Si alguien se pasa conmigo, mi pareja me defiende, aunque no se lo pida»). En la Tabla III pueden consultarse los resultados de la prueba Z y la distribución de cada una de las variables.

En 18 de los 20 casos en los que la prueba Z revela la existencia de diferencias significativas, el alumnado del instituto muestra un grado de acuerdo significativamente superior en comparación con el alumnado universitario. Los dos casos en los que esto ocurre a la inversa hacen referencia a la igualdad («Mis opiniones son tan importantes como las de mi pareja») y la comunicación en la pareja («Nuestra relación se basa en la comunicación. Nos escuchamos»). En cada una de estas variables, la población universitaria se manifiesta de acuerdo en más de un 10\% respecto a la del instituto. En la medida en la que la edad, la experiencia y la formación son mayores, están algo más equilibradas las relaciones de poder en la pareja. 


\section{Tabla III. Variables con mayores diferencias significativas entre muestras}

ÍTEMS

P18. «No tengo problema en darle las contraseñas de mis redes sociales a mi pareja, si así se

siente más tranquilo/a y confía más en mí».

P8. «Si un miembro de la pareja se preocupa constantemente por saber qué está haciendo el

otro, es una muestra de afecto e interés».

P14. «Si alguien se pasa conmigo, mi pareja me defiende aunque no se lo pida».

P26. «Mi pareja me dice que sería capaz de "cualquier cosa" si le dejara».

P27. «Mis opiniones son tan importantes como las de mi pareja».

1 I: instituto; U: universidad

Fuente: elaboración propia.

\section{Conclusiones y líneas de actuación}

Dado que el objetivo del estudio es diagnosticar cómo se expresan las relaciones de poder en las parejas en centros educativos con presencia de población juvenil de entre 14 y 20 años a partir de las inquietudes de un instituto de educación secundaria, la atención de este trabajo se ha dirigido principalmente a la búsqueda de aquellos ítems que muestran diferencias estadísticamente relevantes entre las categorías de las variables sociodemográficas, esto es, sexo y edad. Sin embargo, es necesario señalar que el hecho de que existan diferencias significativas entre las categorías - es decir, que cada submuestra responda de forma distinta-, no equivale a pensar de forma contraria. Más bien, equivale a decir que las personas que se asocian a cada una de las categorías distribuyen sus respuestas de forma diferente, aunque la mayoría de ellas, en ambas categorías, apunte en el mismo sentido sus respuestas.

Los análisis de las diferentes respuestas mostrados en esta contribución permiten vislumbrar algunas conclusiones referidas a la importancia del sexo de cada persona y de la experiencia de relaciones de pareja como variables diferenciadoras de las percepciones y actitudes de la población joven respecto al poder en las mismas. La importancia de la experiencia se manifiesta a través de la edad y el haber tenido o no pareja, pero también por el hecho de pertenecer a la muestra del instituto de educación secundaria o de la universidad, por lo que cabe pensar que pueda haber otras variables implicadas en ella, como podrían ser la formación y una mayor conciencia de la otredad, más allá de los grupos de pertenencia y referencia. Esta mayor conciencia podría estar vinculada al papel de la Universidad en el proceso vital de formación de los individuos.

Los datos analizados a partir de los centros revelan que, en la muestra del instituto, las dimensiones en las que aparece una expresión de actitud diferente a partir de las respuestas obtenidas son, por orden, las de ideales y valores, confianza, poder y hábitos y costumbres. Esta información, así como el orden (generado a partir del número de variables en los que la prueba $Z$ revela la existencia de diferencias significativas entre sus categorías), permite observar que estos son los elementos que se encuentran en tensión entre el alumnado del IES. Esta tensión, como se ha mostrado, aparece respecto a las variables sexo, edad y haber tenido o no pareja.

Sin embargo, si se presta atención a este mismo aspecto respecto a la muestra universitaria, se aprecia que las dimensiones que se vinculan con dicha tensión son, por orden, el poder, la confianza y los hábitos 
y costumbres en las relaciones. A partir de estos resultados puede apreciarse también un cambio en el proceso de socialización del alumnado, ya que se ve como en la población más joven, concretamente la del instituto, el foco está puesto en la idealización de aquello que se espera debe ser una relación de pareja. Mientras, en la población universitaria comienzan a ser más destacables las tensiones por cuestiones más profundas, como son los roles de poder y la comunicación. Comparativamente, las dimensiones que muestran diferencias estadísticamente significativas entre ambas muestras son principal y precisamente, la de poder y la de ideales y valores, lo cual reafirma lo expuesto.

Prestando atención al sexo, ya que es la variable más diferenciadora y común de opiniones según la prueba Z, pueden extraerse algunas conclusiones acerca de los perfiles y roles de hombres y mujeres en las relaciones de pareja. A partir de los datos analizados se observa que las desigualdades se reducen con la edad de las personas entrevistadas, aunque hombres y mujeres mantienen, e incluso acentúan sus elementos diferenciadores. Esto se evidencia al observar que en la población universitaria se encuentra la permanencia de diferencias significativas entre las percepciones y actitudes de hombres y mujeres, aunque en general, como se ha señalado, ambos sexos apuntan sus respuestas hacia un mismo horizonte de acuerdo o de desacuerdo.

No obstante, a partir del análisis de las respuestas de cada uno de los ítems, se aprecia que las chicas dan más importancia a la privacidad mientras que los chicos se la dan a la confianza. Cuando existen diferencias significativas, ellos dicen ofrecer aspectos privados o relacionados con terceras personas a sus parejas si con ello se consigue que la relación funcione. Sin embargo, las respuestas en algunos ítems, en ambos sexos, apuntan a una reproducción de los roles tradicionales. En el caso del ítem «Cuando un miembro de la pareja corrige duramente al otro, lo hace para ayudarle a mejorans, los hombres duplican el grado de acuerdo con el enunciado respecto a las mujeres, en ambas muestras. En el mismo sentido que los estudios de Rubio (2015), las relaciones de pareja entre la población más joven pueden ser, en algunos aspectos, menos democráticas y libres de lo que cabría de esperar en sociedades aparentemente más abiertas y democráticas.

También se observa, en la muestra universitaria, que el 34\% de los hombres está de acuerdo con la afirmación «Necesito estar en constante comunicación con mi pareja, aunque sea por Whats App» (el 20\% de ellas también lo está). Por otro lado, y en el caso de los institutos particularmente, las mujeres duplican el grado de acuerdo respecto a los hombres en el ítem «Si alguien se pasa conmigo, espero que mi pareja me defienda». Ello refuerza la permanencia de roles de género y relaciones de poder tradicionales en la pareja. Como ya han confirmado distintos estudios el uso de los smartphones y de las redes sociales e Internet puede convertirse en una nueva forma de control con cierto poder totalizador al tener una presencia permanente (Bringué y Sádaba, 2009; Velázquez, 2011; Jiménez-Albiar et al., 2012).

De forma menos significativa, la relación entre sexo y orientación sexual permite también alguna observación. De esta forma, los adolescentes y jóvenes varones de menor edad, heterosexuales y que no han tenido pareja, constituyen el grupo donde, con mayor probabilidad, pueden encontrarse valores y comportamientos más sexistas y menos igualitarios. Por otro lado, las jóvenes más mayores que manifiestan otra orientación sexual y que han tenido varias parejas a lo largo de su vida, constituyen el grupo donde, con mayor probabilidad, se pueden encontrar valores y comportamientos menos sexistas y más igualitarios.

Por otra parte, el análisis de los datos plantea un posible enmascaramiento de las relaciones de poder, debido, probablemente, a una concepción romántica de la pareja (Illouz, 2009, 2012), en la que las cuestiones relacionadas con la confianza y el poder son las que muestran diferencias más significativas entre 
chicos y chicas. La comunicación se expresa como uno de los elementos más relevantes en el día a día de las relaciones para quienes han tenido alguna vez pareja. Es destacable el hecho de que para quienes no han tenido relaciones, esta valoración es contraria, lo cual conduce a preguntarnos qué se entiende por comunicación, siendo este interrogante una de las limitaciones en este tipo de investigaciones y que se pretende subsanar en futuras etapas de investigación en relación al proyecto de colaboración con los centros de educación secundaria. Para ello, se planteará la aplicación de metodologías mixtas que permitan un mayor grado de comprensión de los resultados obtenidos.

En resumen, esta contribución recoge información detallada acerca del proceso de investigación y el posterior ajuste para su aplicación en diferentes centros. El diseño, la metodología y el planteamiento global de la investigación están orientados a maximizar las herramientas eficientes para una reflexión crítica en el ámbito educativo, posibilitando espacios a la juventud para la acción y la transformación social. Esta cooperación entre dos centros públicos de educación (un Instituto de Educación Secundaria y la Universidad), está produciendo un conocimiento comprensivo y profundo acerca de cómo viven las alumnas y alumnos las relaciones de pareja, así como cuáles son las narrativas, tensiones, fronteras y marcas de desigualdad que se generan ante la exposición de los resultados obtenidos en el diagnóstico.

Los resultados han cristalizado principalmente en dos efectos. Por un lado, han contribuido a configurar un marco de conocimiento sobre la realidad social del alumnado encuestado en el IES y en la Universidad de Alicante, dada la disposición de datos empíricos cruzados por variables de interés, como son el sexo, la edad, la orientación sexual, el país de nacimiento y el hecho manifestado de haber estado en algún momento en pareja o no. Por otro lado, y coincidiendo con el interés del equipo de investigación del Observatorio Sociológico de la Educación (OBSOEDU), se prioriza la aplicación científica, política y social del trabajo realizado. Este ha dado lugar a la producción de materiales para desarrollar futuros talleres con el alumnado, con la colaboración y orientación del grupo de investigación, mediante la aplicación de técnicas cualitativas, permitiendo profundizar en relaciones concretas. Dichas técnicas cuentan con los inputs obtenidos en la fase de diagnóstico, y con la implicación del personal de los centros, hecho relevante dada su relación cercana con el alumnado.

Los impactos potenciales se circunscriben dentro del territorio local, pero podrían tener efectos a un nivel nacional e internacional dado que, tanto desde la perspectiva europea como global, existe la necesidad de avanzar en el conocimiento científico sobre la forma en que se construyen las identidades en la población joven y sus efectos en las relaciones personales e intersubjetivas. Se considera que los resultados de la investigación en cuestión de género y poder pueden ayudar a comprender procesos similares en otros contextos.

\section{Referencias bibliográficas}

Bauman, Zygmunt (2003). Amor líquido: acerca de la fragilidad de los vínculos humanos. Madrid: Fondo de Cultura Económica de España.

Beck, Ulrich y Beck-Gernsheim, Elisabeth (2001). El normal caos del amor: Las nuevas formas de la relación amorosa. Barcelona: Paidós.

Benjamin, Walter (1973). Discursos interrumpidos I. Madrid: Taurus

Blanco, María Ángeles (2014): "Implicaciones del uso de las redes sociales en el aumento de la violencia de género en adolescentes". Revista Comunicación y Medios, (30), 124-141. DOI: 10.5354/07191529.2015.32375. 
Bourdieu, Pierre (2005): "La dominación masculina revisitada". Archipiélago: Cuadernos de crítica de la cultura, 67, 9-22.

Bringué, Xavier y Sádaba, Charo (2009). La generación interactiva en España. Niños y adolescentes ante las pantallas. Pamplona: Organización Universitaria Interamericana y Telefónica.

Busquet, Jordi y Uribe, Ana Cinthya (2011). El uso de las TIC y la Brecha Digital entre adultos y adolescentes. Primer avance de resultados. Comunicación presentada durante el Congreso Internacional de Educación Mediática y Competencia Digital (en línea). https://bit.ly/2CobpnB, consultado el 28 de abril de 2018.

Castells, Manuel y Subirats, Marina (2007). Mujeres y hombres: ¿Un amor imposible? Madrid: Alianza.

Collins, Randall (2009). Cadenas de rituales de interacción. Barcelona: Anthropos.

Connell, Raewyn (1987). Gender and Power: Society, the Person and Sexual Politics. Stanford: Stanford University Press.

Connell, Raewyn (2002). Gender. Cambridge: Polity Press

Connell, Raewyn (2003). Masculinidades. México: UNAM.

Connell, Raewyn (2015). Gender: in World Perspective. Cambridge: Polity Press.

Cruz Cantero, Pepa y Cobo Bedía, Rosa (1991). Las mujeres españolas: Lo privado y lo público. Madrid: Centro de Investigaciones Sociológicas.

De la Peña, Eva María; Ramos, Esther; Luzón, José María y Recio, Patricio (2011). Sexismo y violencia de género en la juventud andaluza. Resultados y recomendaciones. Madrid: Instituto Andaluz de la Mujer - Junta de Andalucía.

Díaz-Granados, Fernando Iriarte (2007): “Los niños y las familias frente a las Tecnologías de la Información y las Comunicaciones (TIC)". Psicología desde el Caribe, (20), 208-224.

Giddens, Anthony (2004). La transformación de la intimidad: Sexualidad, amor y erotismo en las sociedades modernas. Madrid: Ediciones Cátedra.

Illouz, Eva (2007). Intimidades congeladas: las emociones en el capitalismo. Buenos Aires: Katz.

Illouz, Eva (2009). El consumo de la utopía romántica: El amor y las contradicciones culturales del capitalismo. Buenos Aires: Katz.

Illouz, Eva (2012). Por qué duele el amor. Una explicación sociológica. Buenos Aires: Katz.

Illouz, Eva (2014). Erotismo de autoayuda: “Cincuenta sombras de Grey” y el nuevo orden romántico. Buenos Aires: Katz.

Innerarity, Daniel (2006). El nuevo espacio público. Madrid: Espasa.

Jiménez-Albiar, María Isabel; Piqueras, José Antonio; Mateu-Martínez, Ornela; Carballo, José Luis; Orgilés, Mireia y Espada, José Pedro (2012): "Diferencias de sexo, característica de personalidad y afrontamiento en el uso de Internet, el móvil y los videojuegos en la adolescencia”. Salud y drogas, 12 (1), 61-82.

Kaufmann, Jean-Claude (2015). Identidades. Una bomba de relojería. Barcelona: Ariel.

Leonard Mitchell, Erika (2012). Cincuenta sombras de Grey. Barcelona: Grijalbo. 
Muñiz, María; Cuesta, Pepa; Monreal-Gimeno, Carmen y Povedano, Amapola (2015): "Violencia de pareja online y offline en la adolescencia: el rol de la soledad y del género". Revista sobre la infancia y la adolescencia, (9), 85-97. DOI: https://doi.org/10.4995/reinad.2015.3898.

Rodríguez, María Sol y Vázquez-Portomeñe, Fernando (2015). Estudio empírico sobre la violencia de género. Valencia: Tirant Lo Blanch.

Rubio Castillo, Ana Miguel (2015): "Recensión: Percepción de la violencia de género por la adolescencia y juventud. Delegación del Gobierno para la Violencia de Género (2015). España Ministerio de Sanidad, Servicios Sociales e Igualdad”. Revista Metamorfosis: Revista del Centro Reina Sofía sobre Adolescencia y Juventud, (2), 100-105.

Sobczyk, Rita Aleksandra (2015). Diversidad religiosa en el contexto migratorio andaluz: identidad, fronteras sociales y religión "vivida". Granada: Universidad de Granada.

Turkle, Sherry (2017). En defensa de la conversación. Barcelona: Ático de los libros.

Velázquez, Luz María (2011). Sexting, Sexcasting, Sextorsión, Grooming y Ciberbullying. El lado oscuro de las TIC. Ponencia presentada en el XI Congreso Nacional de Investigación Educativa, Universidad Autónoma de Nuevo León, México (en línea). https://bit.ly/2JhsuE4, consultado el 28 de abril de 2018.

Venegas, Mar (2011): "Un modelo sociológico para investigar las relaciones afectivosexuales". Revista Mexicana de Sociología, 73 (4), 559-589. DOI: http://dx.doi.org/10.22201/iis.01882503p.2011.4.28823.

Venegas, Mar (2018): "El romance adolescente. Un análisis sociológico de la política afectivosexual en la adolescencia". Papers: revista de sociología, 103 (2), 255-279. DOI: http://dx.doi.org/10.5565/rev/papers.2213.

\section{Notas biográficas}

María Jiménez-Delgado es Doctora en Sociología, Profesora y Directora del Departamento de Sociología I e investigadora del Instituto de Investigación de Estudios de Género de la Universidad de Alicante. Recibió el Accésit del XXIII Premio Victoria Kent por la investigación feminista de la Universidad de Málaga (2013) por su trabajo 'Yo soy yo'. Las estudiantes de origen marroquí hablan de sí mismas.

Es directora del Observatorio Sociológico de la Educación de la Universidad de Alicante (OBSOEDU), e Investigadora Principal (IP) en los Proyectos I $+\mathrm{D}+\mathrm{i}$ «Éxito escolar, igualdad e inserción socio-laboral de las mujeres jóvenes de origen magrebí» (2013-2015) e «Identidades de la juventud musulmana: género, educación y ciudadanía» 2017-2019). Sus líneas de trabajo se centran en el género, la igualdad, la inmigración, la multiculturalidad y la educación.

Pablo de-Gracia-Soriano es Graduado en Sociología por la Universidad de Alicante con Premio Extraordinario al Expediente Académico. Realizó el Máster en Dinámicas de Cambio en Sociedades Modernas y Avanzadas en la Universidad Pública de Navarra. Actualmente es Profesor Colaborador Honorífico de la Universidad de Alicante y Doctorando en el Programa de Doctorado de Antropología y Sociología de la Universidad Complutense de Madrid, donde es Becario FPU del Ministerio de Educación y también imparte docencia. Además, está vinculado al Observatorio Sociológico de la Educación, al Theory Group y a la Comisión de la Biblioteca del Departamento de Sociología I de la Universidad de Alicante. Su área 
de investigación principal es la Sociología del Tiempo, aunque ha participado en proyectos relacionados con género, ciudadanía, educación, cooperación, identidades y migraciones. Identificación ORCID: https://orcid.org/0000-0002-4809-4742.

Diana Jareño-Ruiz es Licenciada (Premio Extraordinario al Expediente Académica) y Doctora en Sociología (Doctorado con Mención Internacional y Premio Extraordinario) por la Universidad de Alicante. En esta Universidad es Profesora Ayudante Doctora en el Departamento de Sociología I, impartiendo docencia en las distintas áreas de las Facultades de Ciencias Económicas y Empresariales y de Derecho. Actualmente, es miembro del Observatorio Sociológico de la Educación (OBSOEDU) y participa como investigadora en sendos proyectos de I+D+i de ámbito europeo, nacional y autonómico. Su colaboración en grupos de investigación, sus contribuciones a congresos y sus diferentes publicaciones, se enmarcan dentro de las áreas de familia, población, migraciones, género y educación. Identificación ORCID: https://orcid.org/0000-0001-9482-8017.

Mariano Agustín González-Chouciño es Graduado en Sociología por la Universidad de Alicante (Premio de la Cátedra de Responsabilidad Social de la UA por su Trabajo de Fin de Grado). Realizó el Máster en Desarrollo Local e Innovación Territorial cursado en esta Universidad. Actualmente es Doctorando en el Programa de Doctorado en Empresa, Economía y Sociedad de la Universidad de Alicante y Becario de Prácticas Profesionales en el Instituto Valenciano de la Juventud. Además, es miembro del Observatorio Sociológico de la Educación (OBSOEDU), en el que ha colaborado con contribuciones relacionadas con género, educación y TIC. 